\title{
Kinematic Evolution of Field and Cluster Spiral Galaxies
}

\author{
Bodo L. Ziegler ${ }^{1}$ and Asmus Böhm ${ }^{2}$ \\ ${ }^{1}$ University of Vienna, Department of Astrophysics, Türkenschanzstr. 17, 1180 Vienna, Austria \\ email: bodo.ziegler@univie.ac.at \\ ${ }^{2}$ Institute for Astro- and Particle Physics, Technikerstrasse 25/8, 6020 Innsbruck, Austria \\ email: asmus.boehm@uibk.ac.at
}

\begin{abstract}
We investigate the evolution of the Tully-Fisher relation out to $z=1$ with 137 emission-line galaxies in the field that display a regular rotation curve. They follow a linear trend with lookback time being on average brighter by $1.1 \mathrm{Bmag}$ and $60 \%$ smaller at $z=1$. For a subsample of 48 objects with very regular gas kinematics and stellar structure we derive a TF scatter of $1.15 \mathrm{mag}$, which is two times larger than local samples exhibit. This is probably due to modest variations in their star formation history and chemical enrichment.

In another study of 96 members of Abell 901/902 at $z=0.17$ and 86 field galaxies with similar redshifts we find a difference in the TFR of $0.42 \mathrm{mag}$ in the $B$-band but no significant difference in stellar mass. Comparing specifically red spirals with blue ones in the cluster, the former are fainter on average by $0.35 \mathrm{Bmag}$ and have $15 \%$ lower stellar masses. This is probably due to star formation quenching caused by ram-pressure in the cluster environment. Evidence for this scenario comes from strong distortions of the gas disk of red spirals that have at the same time a very regular stellar disk structure.
\end{abstract}

Keywords. galaxies: spiral - galaxies: evolution

\section{Introduction}

Internal kinematics of galaxies provide important clues about their nature and reveal physically distinct components (e.g. Kalinova et al. 2014). While stars are on colissionless orbits tracing the gravitational potential dominated by dark matter, gas clouds can react faster on subtle effects and may display peculiar motions revealing interaction events. In case of undisturbed spiral galaxies rotation produces a characteristic velocity profile with a steep rise in the inner part turning over to a flat part, the so-called rotation curve RC. In such a virialized state, the maximum velocity $V_{\max }$ provides a good estimate of the total mass of a galaxy and tightly correlates with the luminosity in the TullyFisher relation TFR. Under this assumption the TFR is an important scaling relation to probe quantitatively the evolution of disk galaxies with redshift as applied in several studies before (e.g. Böhm \& Ziegler 2007; Miller et al. 2011). Since stellar kinematics from absorption lines requires a higher $S / N$ most studies of distant galaxies rely on emission line measurements from the warm ionized gas. Here, we report on two applications: a TFR study of distant field galaxies and an investigation of transition objects in the cluster complex Abell 901/2.

\section{Evolution of the Tully-Fisher relation to $\mathrm{z}=1$}

Continuing our previous investigations we expanded our sample towards fainter galaxies with new observations with ESO's VLT using FORS (Böhm \& Ziegler 2014). The whole sample holds high-quality spectra of 238 objects. For 137 disk galaxies we were 


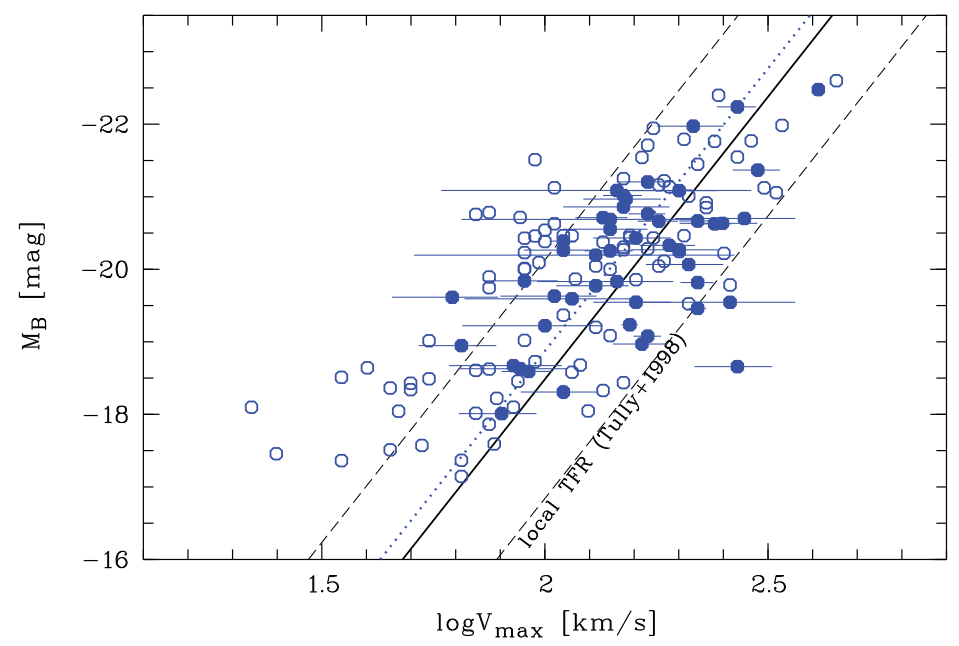

Figure 1. The Tully-Fisher relation of 137 field galaxies with $0.05<z<0.97$ (open symbols) compared to the local relation from Tully et al. 1998 (solid line \& 1- $\sigma$ boundaries dashed). Filled circles represent a subsample of 48 disk galaxies with low morphological asymmetry and high quality rotation curves. Fitting their zeropoint yields the dotted line offset by $0.40 \mathrm{mag}$ towards brighter luminosities.

able to extract RCs and determine $V_{\max }$, while 101 emission-line objects were rejected from our TF analysis due to kinematic distortions. These field spirals are spread like $0.05<z<0.97$ with a median redshift of 0.45 corresponding to lookback times between 0.6 and $7.6 \mathrm{Gyr}$ and $\left\langle t_{\mathrm{lb}}\right\rangle=4.5 \mathrm{Gyr}$. In Fig. 1 we show their TFR in rest-frame $B$-band compared to the local study of Tully et al. (1998). The slope of the distant sample is with $-7.16_{-0.53}^{+0.71}$ similar to the local value of -7.79 . We further create a subsample of 48 objects restricted to have both high-quality RCs and a low structural asymmetry $\left(A_{\text {morph }}<0.25\right.$ in the CAS system of Conselice at al. (2000)) to ensure regularity in both the gas and the stellar component in order to avoid any non-relaxed contribution to the

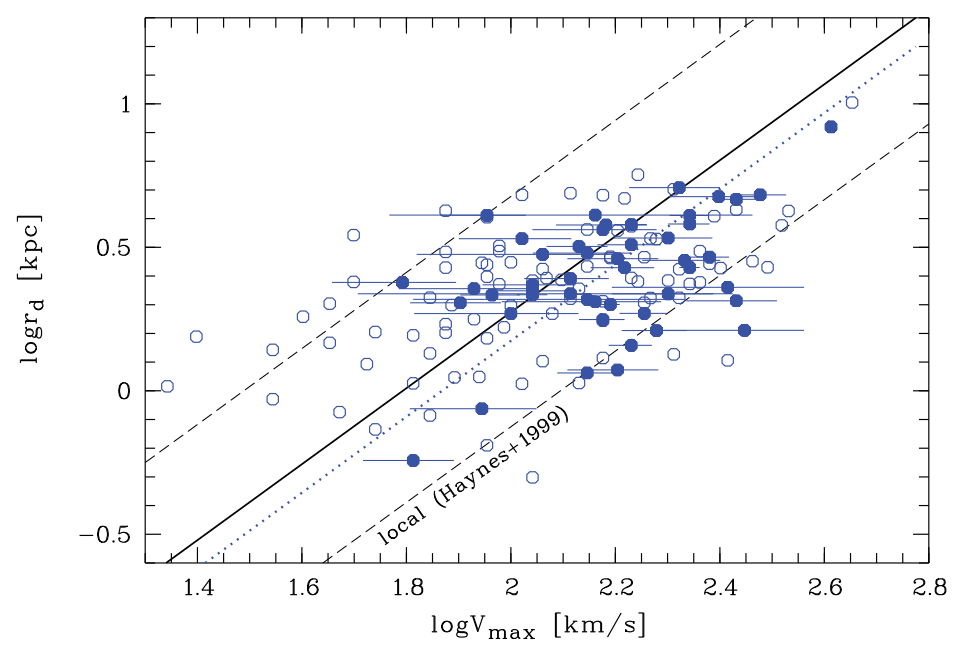

Figure 2. The Velocity-Size relation of the same 137 galaxies like in Fig. 1 this time compared to the local relation from Haynes et al. (1999). On average, distant galaxies have disk scale lengths $r_{d}$ smaller by 0.15 dex than local ones for given $V_{\max }$ (fit indicated by dotted line). 
velocity field. Assuming the local slope this subsample shows moderate brightening of on average $\left\langle\Delta M_{B}\right\rangle(z \approx 0.5)=-0.40^{m}$ and a scatter of $1.15^{m}$, which is two times larger than the local one. This may be attributed to a modest diversity of star formation histories and chemical enrichments of the observed galaxies (Ferreras et al. 2014). Looking at the evolution of the TF residuals from the local relation we fit our whole sample linearly with lookback time and find $\Delta M_{B}=-(3.63 \pm 1.78) \log (1+z)$ albeit with large scatter. Such an increase in luminosity is derived by other studies, too (e.g. Miller et al. 2011), and in accordance with model predictions (Dutton et al. 2011).

We also investigate the Velocity-Size evolution of the same galaxies by measuring their disk scale lengths $r_{d}$ in HST $I$-band images. Although the scatter is rather large, our sample is offset by -0.15 dex to smaller $r_{d}$ for given $V_{\max }$ compared to the local sample of Haynes et al. (1999), see Fig. 2. Note that the local sample was observed in $I$-band, too, and the scale lengths of the distant galaxies were slightly corrected for rest-frame wavelength shift following de Jong (1996). On average, disk sizes decrease by $\Delta \log r_{d}=-(0.59 \pm 0.38) \log (1+z)$ becoming about $60 \%$ smaller at $z=1$, which is again in accordance with models.

\section{Red spirals as stripped transition objects in clusters}

Looking for environmental effects we examine galaxies in the multiple cluster system Abell 901/902 at $z=0.17$ (Gray et al. 2009). An observational program with ESO's VLT using VIMOS targeted $\sim 200$ emission-line galaxies in the cluster complex with tilted slits placed along the major axis (PI A. Böhm, see Bösch et al. (2013a)). A kinematic analysis was possible for 96 cluster members and 86 field spirals with a similar average redshift $(0.12<z<0.38)$. However, many objects don't exhibit a regular RC but distorted kinematics. To quantify the degree of distortion we introduce similar to Dale et al. (2001) an RC asymmetry index $A_{\mathrm{RC}}$ based on differences of the approaching and receding arms. We find an average value of $\left\langle A_{\mathrm{RC}}\right\rangle=17 \%$ in the field, while distortions are more frequent in the cluster, yielding $\left\langle A_{\mathrm{RC}}\right\rangle=25 \%$. Restricting to objects with highquality RCs for a TF analysis we find that cluster members are on average modestly fainter in rest-frame $B$-band by $\Delta M_{B}=0.42 \pm 0.15^{m}$. However, there is no significant difference in the stellar TF with $\Delta \log M_{*}=0.00 \pm 0.07$.

Further, we concentrate on red spirals that were defined by Wolf et al. (2009) to have red colors similar to red-sequence galaxies but (spiral) disk morphology and significant star formation rates (SFRs), albeit lower than typical for blue spirals. Those red spirals with regular RCs are on average fainter in the $B$-band TFR by $\Delta M_{B}=0.35 \pm 0.12^{m}$ and show a slight difference in the stellar TF by $\Delta \log M_{*}=0.06 \pm 0.05$ compared to bluecloud cluster members (Bösch et al. 2013b). These results may be explained by a recent quenching of SF for red spirals caused by ram-pressure stripping in the cluster removing gas. This interpretation is strengthened by Fig. 3: many red spirals show strong rotation curve asymmetries combined with an undisturbed stellar morphology. The only clusterspecific interaction process which affects the gas disk but not the stellar morphology is ram-pressure (Kronberger et al. 2008). Thus, our analysis adds evidence that red spirals are transition objects between field blue-cloud and cluster lenticular galaxies.

\section{Acknowledgements}

Based on ESO PID 081.B-0107, 384.A-0813 and other programs. AB acknowledges support by the Austrian Science Foundation FWF (grants P19300-N16 and P23946-N16). BZ thanks SOC and LOC for a very nice symposium. 


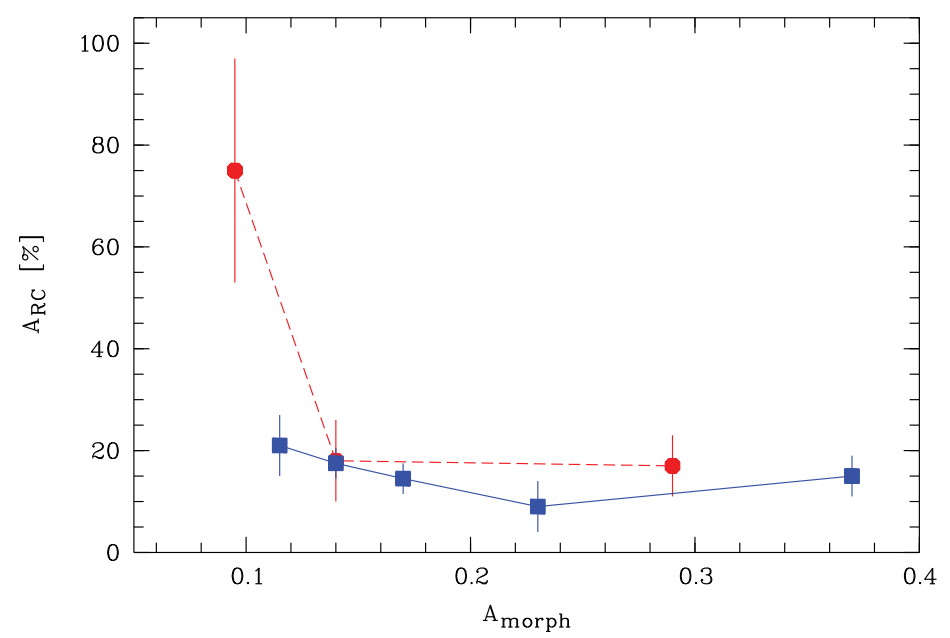

Figure 3. Rotation curve asymmetry $A_{\mathrm{RC}}$ versus morphological asymmetry $A_{\text {morph }}$ for red spirals (circles) and blue spirals (squares) in the cluster system A901/902. Average error-weighted values have been computed for bins holding 9 (red) and 12 objects (blue), respectively. A large fraction of red spirals show strong rotation curve asymmetries, i.e. disturbed gas kinematics, combined with undistorted stellar structure (i.e. low $A_{\text {morph }}$ ). This can best be explained by ram pressure due to the intra-cluster medium.

\section{References}

Böhm, A. \& Ziegler, B., 2007, ApJ, 668, 846.

Böhm, A. \& Ziegler, B., 2014, A\&A A, in prep.

Bösch, B., Böhm, A., ..., Ziegler, B., et al., 2013a, A\&A 549, A142

Bösch, B., Böhm, A., ...., Ziegler, B., et al., 2013b, A\&A 554, A97

Conselice, et al., 2000, ApJ, 529, 886

Dale, et al., 2001, AJ, 121, 1886

de Jong, R. S. 1996, å, 313, 377

Dutton, et al., 2011, MNRAS, 410, 1660

Ferreras, et al., 2014, MNRAS, 437, 1872.

Gray, et al., 2009, MNRAS, 393, 1275.

Haynes, et al., 1999, AJ, 117, 1668

Kalinova, et al., 2014, A\&A, in prep.

Kronberger, et al., 2009, å, 483, 783

Miller, et al., 2011, ApJ, 741, 115

Tully, et al., 1998, AJ, 115, 2264

Wolf, et al., 2009, MNRAS, 393, 1302 